J3eA, Journal sur l'enseignement des sciences et technologies de l'information et des systèmes,

Volume 3, Hors-Série 1, 15 (2004)

DOI : http://dx.doi.org/10.1051/bib-j3ea:2004615

(C) EDP Sciences, 2004

Fusion multi capteurs dans un capteur de chute :conception et résultats

N. Noury ${ }^{1,3}$, P. Barralon ${ }^{1}$, G. Virone ${ }^{1}$, P. Rumeau ${ }^{2}$ et P. Boissy ${ }^{3}$

${ }^{1}$ TIMC-IMAG, UMR CNRS 5525, F-38700 La Tronche, France

${ }^{2}$ Hôpital Charles Foix, Ivry-sur-Seine, France

${ }^{3}$ Centre de Recherches sur le Vieillissement, Université de Sherbrooke, Québec 


\title{
Fusion multi capteurs dans un capteur de chute : Conception et résultats
}

\author{
Norbert Noury $^{1,3}$, Pierre Barralon ${ }^{1}$, Gilles Virone ${ }^{1}$, \\ Pierre Rumeau ${ }^{2}$, Patrick Boissy ${ }^{3}$ \\ ${ }^{1}$ TIMC-IMAG - UMR CNRS 5525 - 38700 La Tronche, France \\ ${ }^{2}$ Hôpital Charles Foix - Ivry sur Seine - France \\ ${ }^{3}$ Centre de Recherches sur le Vieillissement - Université de Sherbrooke, Québec
}

\section{INTRODUCTION}

\section{A. L'intérêt du capteur}

La chute est la première cause de décès traumatique après 65 ans, c'est également une cause majeure de séquelles. Par exemple, après une fracture du col du fémur un tiers des patients décède dans l'année, un tiers garde des séquelles et un tiers seulement récupère son niveau de fonctionnement antérieur. La chute a également des conséquences psychologiques tout aussi invalidantes : la peur de retomber engage un déclin fonctionnel des sujets chuteurs dans $30 \%$ des cas, qui se termine rapidement par un placement en institution.

La chute est un événement fréquent avec l'âge. Après 65 ans, 30\% des personnes âgées vivant à domicile chutent de façon significative au moins une fois par an, après 75 ans, 32 à $42 \%$ [NEV]. Après une première chute, la probabilité de retomber est multipliée par 20 [DAR]. Les conséquences économiques de la chute sont considérables. Elles sont estimées à 2 milliards d'Euros en France, dont la moitié pour les soins, hospitalisations et prescriptions, et l'autre moitié pour les frais de séjours en maison de repos, et séances de rééducation. De part les coûts financiers (10000 $€$ pour une fracture du col du fémur ! $1500 €$ pour une maison de retraite peu onéreuse) mais également du pretium doloris (douleur physique, isolement familial, hospitalisation...) la chute est le premier problème de santé publique des personnes âgées [KOS96].

Les conséquences de la chute doivent être dépistées au plus vite pour éviter des complications douloureuses et coûteuses inutiles (syndrome de la tortue, rhabdomyolyse, complications du décubitus prolongé) qui grèvent le pronostic fonctionnel. En effet une intervention plus rapide des secours réduit le niveau de coût des soins, le besoin en rééducation et favorise un retour précoce au domicile. Egalement on constate que les dispositifs actuels d'alarme et de secours ne prennent pas en compte les chutes causées par une perte de connaissance ou un trouble de la conscience. Dans le cadre des formes évoluées de la maladie d'Alzheimer (problème principal du maintien à domicile des plus de 70 ans) le patient n'a souvent pas les ressources intellectuelles suffisantes pour mettre en place une stratégie d'appel au secours autre que celle d'appeler de là où il est tombé.

Malgré la fréquence clinique pertinente qui les caractérise, les chutes sont un événement statistiquement rare ; l'absence de chutes a basse énergie enregistrées à disposition des chercheurs, va nous obliger à nous baser pour nos études de détection sur un pré-modèle empirique défini par une fusion prenant en compte la connaissance qualitative expérimentale clinique du développement d'une chute et les connaissances sur les facteurs causaux.

\section{B. Principes pour la détection automatique de chutes}

La chute est d'abord un trouble de la marche et des transferts. Le déplacement volontaire est une suite coordonnée d'équilibres instables et de déséquilibres contrôlés permettant une économie énergétique. L'équilibre dynamique ainsi défini peut être rompu par un événement modifiant les paramètres du déplacement (attention, contrôle cognitif, environnement évolutif, syncope). Cette chute résultera dans un déplacement du corps vers le bas avec une vitesse proportionnelle à la durée de la chute puisque l'accélération de la pesanteur reste constante. Il s'agit d'une chute "à basse énergie ", contrairement aux accidents du sport par exemple. Chez la personne âgée, 95\% des cas de chutes sont de ce type.

Les dispositifs classiques de téléalarme ("alarme sociale"), nécessitent en général l'intervention de la personne : il s'agit d'un bouton d'appel, porté en médaillon ou au poignet, qui lorsqu'il est activé met la personne en communication (interphonie) avec un opérateur distant. Ces dispositifs souffrent de déclenchements abusifs (faux positifs) mais également de situations d'urgences non traitées par défaut d'actionnement (faux négatifs), soit que l'appareil n'est pas porté, soit que la personne se trouve dans l'incapacité de l'activer. Il y a donc place pour un dispositif autonome, qui puisse détecter la chute de manière fiable puis transmettre cette information, de préférence par des moyens sans fils s'il est embarqué.

La détection de la chute peut faire appel à des capteurs embarqués sur la personne (endocapteurs), ou au contraire à des capteurs situés dans l'environnement (exocapteurs), mais on peut aussi imaginer de combiner les 2 méthodes.

La détection par exocapteurs fait largement appel aux techniques d'intelligence artificielle et dépend toujours d'un apprentissage, supervisé ou non, qui est souvent très long ce qui rend ces méthodes inapplicables dans l'environnement normal du sujet. Les dispositifs destinés à être embarqués sur la personne se basent principalement sur la détection du choc au sol, ou de la posture horizontale.

Ge Wu de l'Université de Vermont [WU000] analyse les vitesses linéaires verticales et horizontales du buste pour rechercher une signature de la chute par comparaison avec d'autres activités normales de la vie quotidienne. Il place pour cela plusieurs cibles optiques sur la personne, observées à l'aide d'une caméra. Il démontre qu'en activité 
normale ces vitesses restent en dessous du seuil de $1 \mathrm{~m} / \mathrm{s}$ alors que lors d'une chute elles dépassent simultanément cette limite. Il conclut que ses résultats permettent d'envisager le développement d'un dispositif qui détecterait la chute avant l'impact au sol et pourrait ainsi déclencher un système d'amortissement de la chute (Airbag).

Bob Kemp de l'Université de Leiden au Pays-Bas [KEM98] a développé un dispositif qui intègre 3 accéléromètres et 3 magnétomètres qui lui permettent de déterminer à quelques degrés près l'orientation en $3 \mathrm{D}$. Il réparti 10 capteurs de ce type sur la personne et transmet leurs informations par télémétrie dans un PC qui se charge du traitement en temps réel et du diagnostic de chute.

Gareth William de l'Université de Bangor-Pays de Galles [WIL98] met en œuvre un détecteur de chocs piézoélectrique et un interrupteur "tilt" au mercure pour l'indication posturale (position allongée). Un microcontrôleur gère les signaux provenant de ces 2 capteurs et communique par moyen $\mathrm{HF}$ avec un combiné téléphonique qui détient la base de connaissance chargée de décider s'il s'agit d'un "événement de chute" dans le cas où la personne parvient à se relever d'elle-même, ou bien d'une "alarme de chute" dans le cas contraire.

\section{Le Capteur de chute développé pour notre étude}

Le capteur de chute que nous avons développé intègre sur un même support (Figure 1) 3 accéléromètres disposés orthogonalement et un micro-contrôleur qui détermine l'inclinaison du corps puis élabore de manière autonome l'information de chute [NOU01].

Nous avons fait 2 hypothèses simplificatrices : nous observons uniquement la cinématique du tronc et nous nous limiterons dans un premier temps à l'observation dans le plan des déplacements frontaux de la personne. En conséquence nous plaçons un dispositif unique dans le plan « para sagittal» de la personne, sous l'aisselle.

En cas de chute, l'appareil émet dans un premier temps un signal sonore. Le porteur dispose d'un bouton poussoir si il veut désactiver cette alarme en cas de «fausse chute». Si dans un délai imparti il ne l'a pas fait, alors un message d'alarme est émis, par une liaison sans fils, vers un ordinateur PC qui se charge de répercuter l'alarme au centre de télésurveillance.

\section{METHODE DE MESURE}

\section{A. Architecture physique du capteur de chute}

Le capteur intègre principalement 2 accéléromètres biaxes, et un microcontrôleur qui exécute un programme spécifique (Figure 2).

Le micro-contrôleur AT90LS8535 (ATMEL) possède une architecture RISC avec une unité de calculs sur 8 bits. Il est cadencé à $4 \mathrm{MHz}$ et alimenté sous une tension réduite de 3 Volts afin de réduire sa consommation globale. Sa mémoire programme de type Flash, d'une capacité de $8 \mathrm{~K}$ octets, est accessible directement par une liaison série synchrone (SPI) ce qui simplifie la mise à jour de son programme. Il possède une liaison série asynchrone qui permet la communication vers un ordinateur soit directement soit au travers d'un modem sans fils.

Par l'intermédiaire de ses compteurs internes, le microcontrôleur mesure les signaux provenant de 2 accéléromètres bi-axes.

\section{B. Principe de l'accéléromètre}

L'accéléromètre mesure l'accélération dynamique, due aux chocs et vibrations, en même temps que l'accélération statique, due à la force de gravitation. Notre capteur de chute intègre l'accéléromètre ADXL202 (Analog Devices) qui est basé sur un principe capacitif. Une poutre suspendue (masse sismique) est solidaire de l'armature centrale d'un condensateur plan différentiel. Les déplacements de la poutre se traduisent par une variation de la distance entre les armatures et donc une variation de la capacité du condensateur qui peut être mesurée dans un circuit électrique.

Le circuit ADXL202 intègre 2 accéléromètres de ce type disposés orthogonalement dans le plan de la puce (Figure 3). En sortie, le composant produit 2 types de signaux : deux tensions continues correspondant à l'accélération vue par l'axe (XFILT, YFILT), et 2 signaux carrés dont le rapport cyclique est proportionnel à l'accélération appliquée à l'axe (X OUT, Y OUT).

\section{Méthode d'acquisition de données}

Les signaux numériques traduisent l'accélération en rapport cyclique (Figure 4). Les durées T1 et T2 sont mesurées grâce aux compteurs 16 bits du micro-contrôleur. On atteint une résolution de $0,2 \%$ sur la mesure d'accélération.

\section{Algorithmes}

Les évènements à détecter ont des périodes assez longues (500 à $1000 \mathrm{~ms}$ ) aussi on a choisit d'échantillonner les signaux avec une période de $50 \mathrm{~ms}(20 \mathrm{~Hz})$.

La tâche de calcul est composée de 6 phases : acquisition, extraction, classification, filtrage, décision et communication.

La tâche d'acquisition est chargée de numériser les signaux provenant des accéléromètres et de retirer le bruit d'acquisition par des filtres passe-bas numériques coupant au-dessus de $10 \mathrm{~Hz}$.

La tâche d'extraction est en charge d'évaluer l'inclinaison et la vitesse d'inclinaison du buste (capteurs « logiciels » $\alpha$ et $d \alpha / t)$.

La tâche de classification se charge de passer au niveau symbolique les paramètres d'inclinaison $\left(\bar{\alpha}^{-}\right)$et de vitesse d'inclinaison $(d \bar{\alpha} / d t)$ :

$\bar{\alpha} \in\{$ Debout, penché, couché\}

$d \bar{\alpha} / d t \in\{$ Faible, moyenne, élevée $\}$ 
La tâche de filtrage opère un lissage sur les variables symboliques par moyenne glissante afin d'éliminer les décisions intempestives dues aux effets de bords.

La tâche de décision cherche à reconnaître, dans les séquences d'évènements, des scénarios prédéterminés. Pour cela elle met en œuvre des agents logiciels indépendants qui travaillent sur une même séquence de données. Par exemple, la séquence " Debout-vitesse élevéeallongé » est un scénario de chute, alors que la séquence "Deboutvitesse moyenne-allongé » est un scénario d'allongement.

La tâche de communication émet un message codé correspondant au scénario détecté. Pour réduire le nombre de communications, et donc la consommation de l'ensemble, il n'y a émission que si le scénario est différent du précédent scénario détecté.

Le temps total nécessaire à la tâche de calcul est de l'ordre de $10 \mathrm{~ms}$, aussi le microcontrôleur est mis en veille pendant les $40 \mathrm{~ms}$ qui restent ce qui contribue encore à réduire la consommation de l'ensemble.

\section{RESULTATS ET DISCUSSIONS}

\section{A. Modèles de chutes}

Il existe une très grande variété de chutes. Nous avons démarré notre étude par le cas le plus évident celui de la chute en avant (Figure 5). On constate plusieurs séquences : début en posture verticale, vitesse élevée du buste, impact au sol, et posture allongée après l'impact.

Un algorithme basé uniquement sur la détection de la composante d'accélération horizontale peut facilement être pris en défaut par exemple par un trébuchement (Figure 6). Le capteur, placé sous l'aisselle gauche, détecte donc une position allongée : toutes les conditions de la chute sont remplies, il y a donc une fausse décision (faux positif). Or, après quelques pas rapides vers l'avant, la personne commence à ralentir et à reprendre sa stabilité, sa posture redevient verticale, elle reprend une marche normale. On constate qu'il faut prendre en compte ce qui se passe audelà de la détection préliminaire de chute. Un algorithme simpliste peut également être mis en défaut dans le cas de la syncope se terminant en position assise (Figure 7). Dans ce cas toutes les conditions de la chute ne sont pas remplies et on a une fausse décision (Faux négatif).

\section{B. Méthodologie}

Les tests ont été menés au laboratoire sur la posture du Centre de Recherche sur le Vieillissement à Sherbrooke, Québec. L'étude a porté sur 10 sujets dont 8 femmes. La taille moyenne de ces sujets est $172,6 \pm 6,9 \mathrm{~cm}$ et leur poids moyen est $61,2 \pm 4,9 \mathrm{~kg}$. L'age moyen des sujets est de 21,2 ans (compris entre 20 et 24) : on a choisi de recruter des personnes jeunes car les essais demandent beaucoup d'efforts et d'endurance au niveau physique.

Lors des essais, les sujets portaient le capteur de chute placé sur une ceinture sous l'aisselle gauche. En outre ils portaient de multiples cibles optiques suivies par des caméras (OPTOTRAC) et même des accéléromètres ADXL210 $( \pm 10 \mathrm{G})$ placés en des points complémentaires.

Les essais étaient effectués sur un matelas. Enfin, les sujets effectuaient les essais à leur propre rythme sans contrainte de temps.

Le système d'acquisition était constitué d'une station de gestion et d'enregistrement d'OPTOTRAC et d'une station d'enregistrement des données provenant d'autres capteurs.

L'analyse des données a été menée seulement sur les données provenant du capteur de chute.

\section{Le protocole d'essai}

Il existe un grand nombre de types de chute, aussi on a choisi de se limiter à celles qui peuvent nous apprendre le plus. La liste complète de 15 conditions de chute ou non chute (avec leur code de test) à tester était la suivante :

- Catégorie chute en arrière (simulation d'une glissade)

1. se terminant en position assise (x ITBSP xxx)

2. se terminant en position allongée ( $\mathrm{x}$ ITB1B $\mathrm{xxx}$ )

3. se terminant en position allongée latérale ( $\mathrm{x}$ ITB1C $\mathrm{xxx}$ )

4. chute avec récupération (non chute) (x ITB1D xxx)

- Catégorie chute en avant (simulation d'un trébuchement)

5. avec protection des bras (x ATF2A xxx)

6. se terminant en position allongée à plat $(\mathrm{x} A T F 2 B \mathrm{xxx})$

7. avec rotation se terminant en position allongée ( $\mathrm{x}$ ATF2C $\mathrm{xxx}$ )

8. chute avec récupération (x ATF2D xxx)

- Catégorie syncope (perte connaissance)

9. se terminant en positon assise contre le mur (x SVF3A $\mathrm{xxx}$ )

10. se terminant en position allongée à plat ( $\mathrm{x}$ SVF3B $\mathrm{xxx}$ )

11. avec rotation se terminant en position allongée latérale( x SVF3A xxx)

- Catégorie neutre (situation dite "normale »)

12. s'asseoir sur lit et se coucher ( $\mathrm{x}$ NNC4A xxx)

13. marcher, se pencher, se mettre à genou et attacher son soulier (x NNC4B xxx)

14. marcher et faire une collision latérale avec le mur ( $\mathrm{x}$ $\mathrm{NNC4C} \mathrm{xxx}$ )

15. s'asseoir sur une chaise à partir de la position debout ( $\mathrm{x}$ NNC4D xxx)

Pour chaque condition, on a réalisé 5 essais, soit au total 75 essais par sujet. On ne pouvait pas faire plus dans un temps limité, afin de ne pas exposer les sujets à des risques physiques inutiles.

\section{Signaux obtenus}

Les données enregistrées ont été exploitées sous le logiciel de calcul Matlab pour obtenir des tracés interprétables. Un exemple de la trame de données envoyée lors d'un essai de la condition 1 (chute en arrière se terminant allongée à plat) est donné figure 8 . 


\section{E. Bilan de performance}

A partir des 750 enregistrements effectués sur les 10 sujets nous avons effectué un premier bilan des « Faux positifs » (détections erronées - Figure 1) et des « Faux négatifs » (non détections erronées - Figure 2).

La spécificité du capteur est $\mathrm{SP}=0,83$ et sa sensibilité est $\mathrm{SE}=0,79$. Le taux de bonnes détections (Vrais positifs-VP et vrais négatifs-VN) est de $81 \%$ alors que le taux de fausses détections (Faux positifs-FP et Faux négatifs-FN) est de $19 \%$.

D'après ces deux résultats on distingue deux cas critiques de dysfonctionnement du capteur :

- Syncope se terminant assise (faux négatif)

- Chute en avant avec récupération (faux positif)

Nous travaillons actuellement à l'amélioration de ces résultats en mettant en œuvre des algorithmes de décision à l'aide de logique floue et de fusion de décisions à l'aide de méthodes non paramétriques.

\section{CONCLUSIONS ET PERSPECTIVES}

L'étude de la chute peut être menée dans plusieurs directions :

- La détection de la chute vise à déclencher une mise en place rapide des secours afin de limiter les conséquences de la chute ou bien une détection précoce de la chute pourrait permettre de déclencher un dispositif de protection (ex Airbag).

- La protection en cas de chute consiste à concevoir des prothèses de protection qui ne sont pas toujours tolérées par le patient.

- La prédiction de la chute viserait à détecter les signes avant coureur de la chute et à mettre en place une stratégie préventive (médication, adaptation du domicile) ou de protection active (Airbags).

Les résultats préliminaires obtenus avec notre capteur semblent conforter l'idée que l'on peut détecter la chute en observant uniquement la cinématique du tronc. On obtient un taux de performance de l'ordre de $84 \%$ qui est encourageant mais reste insuffisant devant l'objectif de détection de la chute avec $100 \%$ de réussite.

Il faut encore modérer ces résultats car nos mesures ont été effectuées sur une population limitée, que les chutes étaient toutes simulées et que les sujets étaient tous jeunes et en bonne santé. Or il y a une baisse de la mobilité et une plus grande latence avec l'âge.

Dans un premier temps nous n'avons pas encore exploité les informations délivrées dans le plan latéral et on peut espérer que cela permettra d'affiner la détection de la chute latérale depuis la posture verticale. On disposera alors de l'information supplémentaire de la posture du corps lorsque la personne est allongée.

Nous travaillons surtout à l'amélioration du processus de décision en faisant appel à des traitements plus élaborés sur les signaux. D'une part par une décision sur des données floues en faisant appel à la théorie des possibilités, d'autre part en s'appuyant sur une décision pondérée en faisant appel à la théorie de l'évidence.

\section{REMERCIEMENTS}

Nous tenons à remercier le ministère français de l'enseignement supérieur et de la recherche pour le financement du projet de "Vêtement de Télé-Assistance Médicale Nomade " (VTAMN). Nous remercions également le Ministère Français des affaires étrangères et le Ministère Québécois des relations internationales qui ont co-financé notre séjour au centre de recherche sur le vieillissement à l'Université de Sherbrooke.

\section{BIBLIOGRAPHIE}

[DAR] P. Dargent-Molina, G. Bréart,“Epidémiologie des chutes et des traumatismes liés aux chutes chez les personnes âgées », Epidémiologie et Santé Publique, 1995.

[DEP01] Y. Depeursinge, J. Krauss, M. El-Khoury, "Device for monitoring the activity of a person and/or detecting a fall", US Patent 6201 476, Mar. 13, 2001

[KEM98] B Kemp, A Janssen, B Van Der Kamp, "Body position can be monitored using miniature accelerometers and earth magnetic field sensors", Electroencepha. Clinical Neurophysiology, vol 109, pp. 484-488, 1998.

[KOS96] K Koski, H Luukinen, "Physiological factors and medications as predictors of injurious falls by elderly people: a prospective population based study" Age and Ageing, vol 25, pp 29-38, 1996

[NEV] MC Nevitt, SR Cumming, "Risk factors for recurrent non syncopal falls. A prospective study", JAMA, 261, pp 2663-2668.

[NOU00] N. Noury, Rapport d'activité de Norbert NOURY pour l'obtention de l'HDR, Chapitre 3.2, pp. 51-55

[NOU00] N. Noury, T. Hervé, V. Rialle, G. Virone, E. Mercier, Monitoring behavior in home using a smart fall sensor and position sensors, IEEE-EMBS «Microtechnologies in Medicine \& Biology», Lyon-France, Oct 2000, pp 607-610

[NOU01] N. Noury, «Détecteur de chute d'une personne», Brevet $\mathrm{N}^{\circ} 01 / 12046$ du 18 Septembre 2001, Université Joseph Fourier de Grenoble

[NOU02] N. Noury, A smart Sensor for the remote follow up of activity and fall detection of the elderly, in Proc. IEEEMMB2002, Madison-USA, May 2002, pp 314-317.

[WIL98] G Williams, $\mathrm{K}$ Dougthy, $\mathrm{K}$ Cameron, DA Bradley, "A smart fall and activity monitor for telecare applications", in proceedings of 20th International conference IEEE-EMBS, Hong-Kong, Oct 1998, pp 11511154.

[WU000] Ge Wu, "Distinguishing fall activities from normal activities by velocity characteristics", Journal of Biomechanics 33 (2000), 1497-1500 


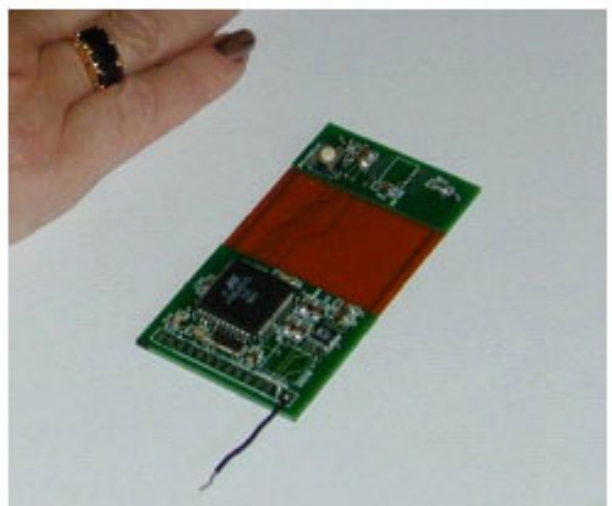

Fig.1. Prototype de capteur de chute.

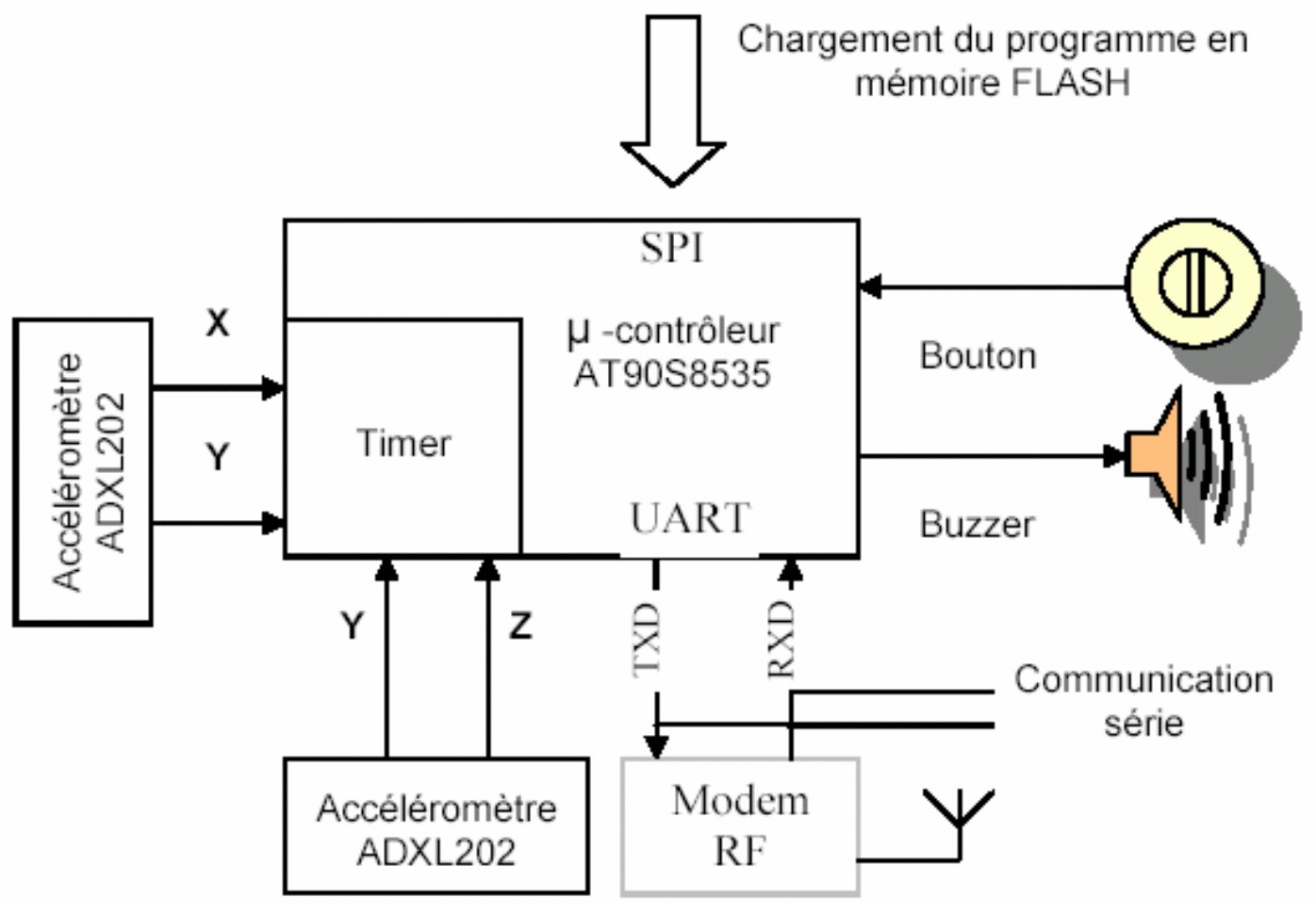

Fig.2. Architecture du capteur de chute. 


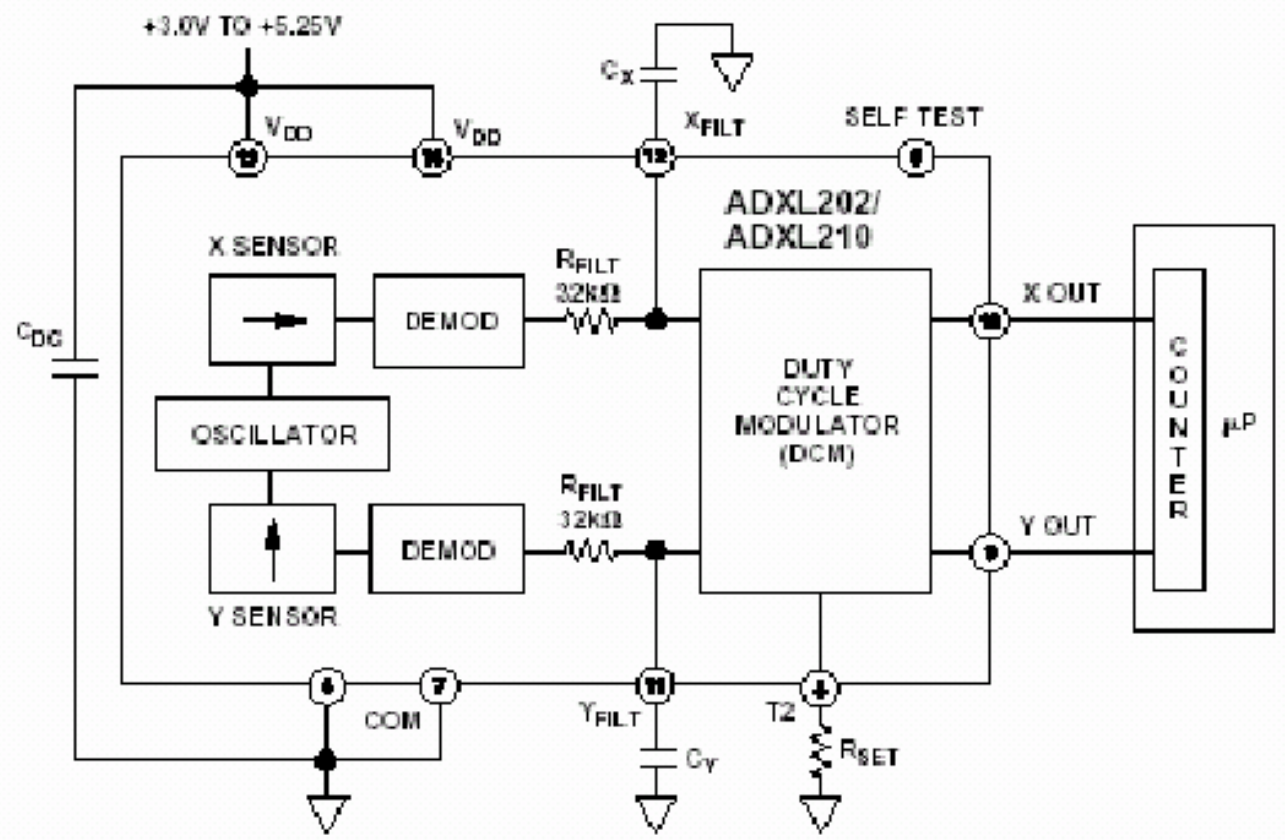

Fig.3. Le schéma fonctionnel de ADXL202.

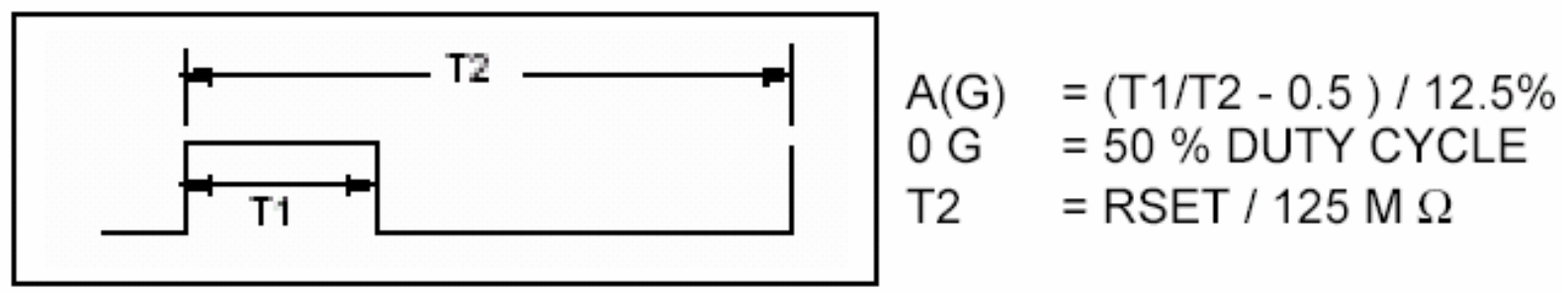

Fig.4. La forme du signal Xout et Yout.

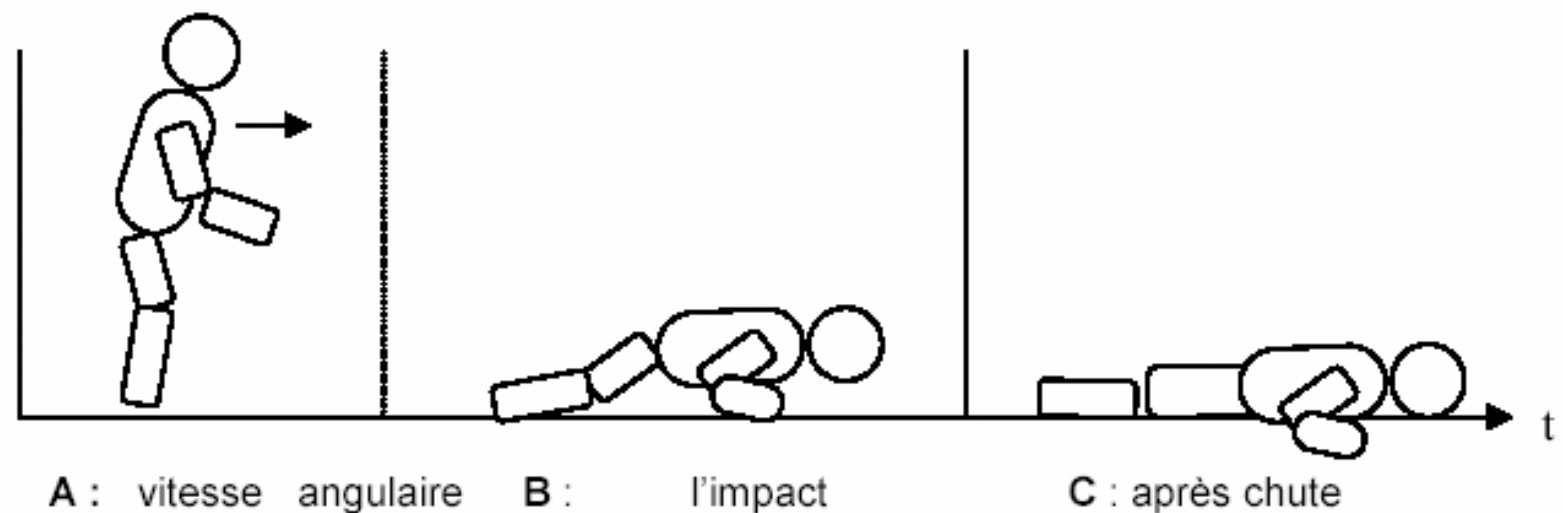
élevée

Figure 5 : Décomposition d'une chute en avant en 3 sous- événements. 


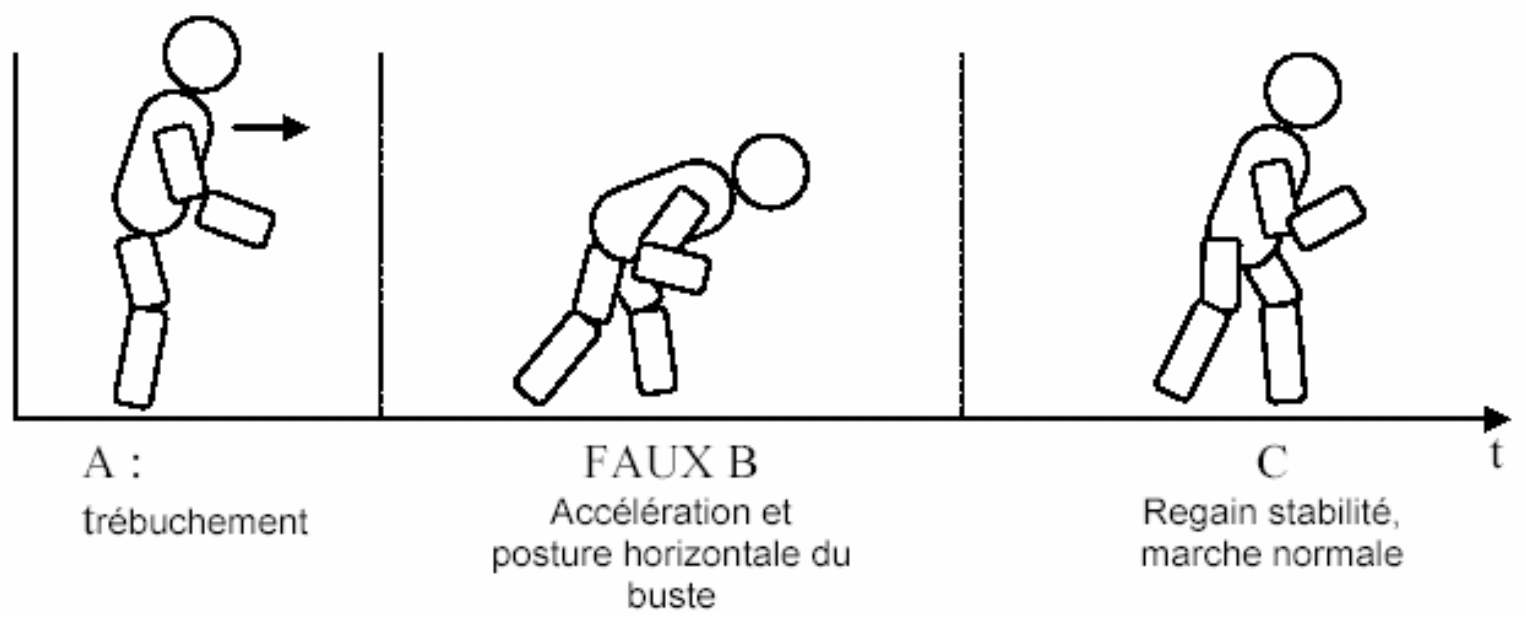

Fig.6. Décomposition d'un trébuchement en 3 sous événements.

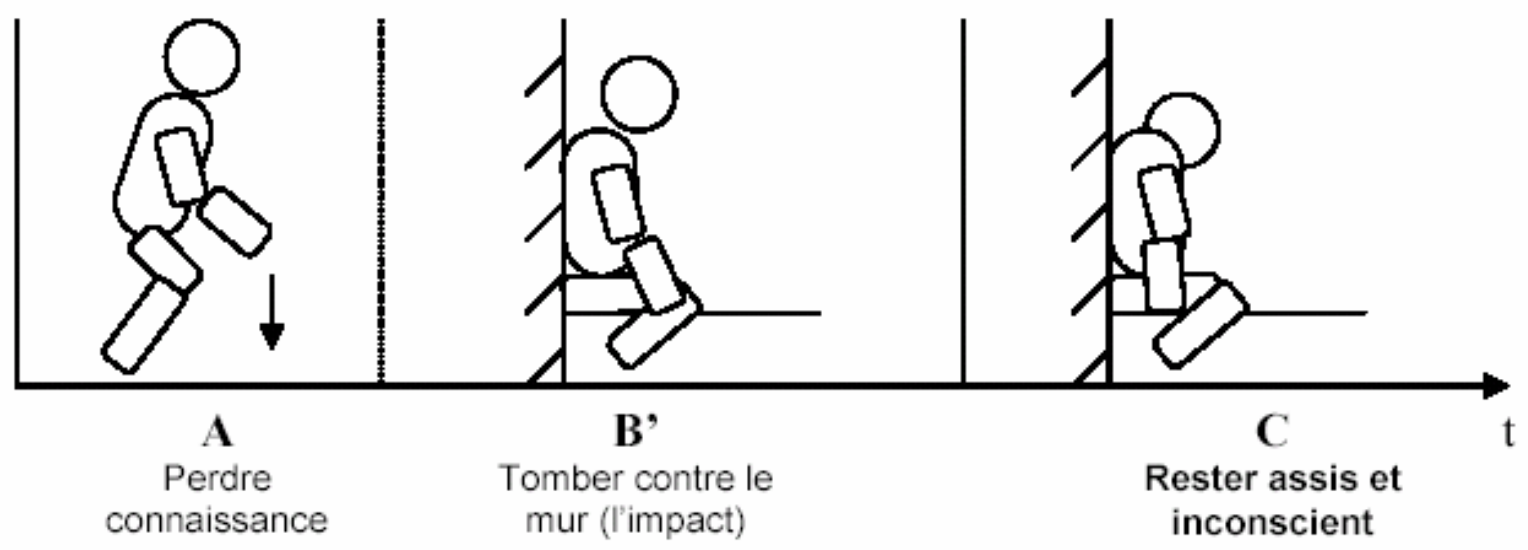

Fig.7. Décomposition d'une syncope en 3 sous événements. 